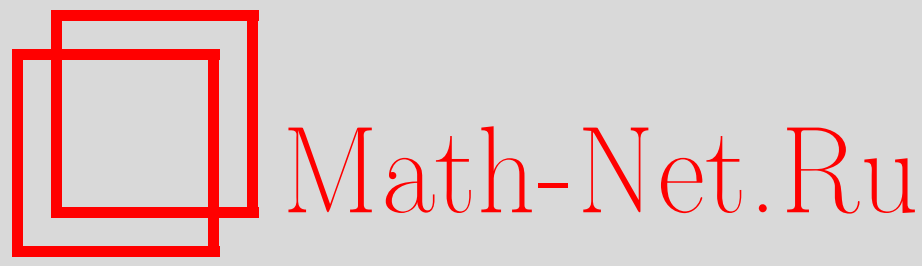

Н. А. Славнов, Асимптотические разложения для корреляционных функций одномерных бозонов, ТМФ, 2013, том 174, номер 1, 125-139

DOI: https://doi.org/10.4213/tmf8363

Использование Общероссийского математического портала Math-Net.Ru подразумевает, что вы прочитали и согласны с пользовательским соглашением http://www . mathnet.ru/rus/agreement

Параметры загрузки:

IP : 54.198 .67 .100

26 апреля 2023 г., 15:47:29

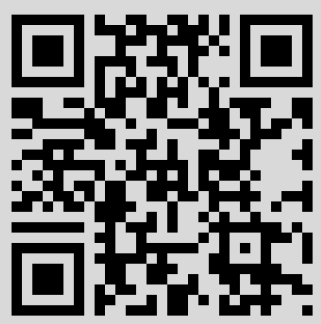




\title{
ФИЗИКА
}

Том 174, № 1

январь, 2013

(C) 2013 г.

Н. А. Славнов*

\section{АСИМПТОТИЧЕСКИЕ РАЗЛОЖЕНИЯ ДЛЯ КОРРЕЛЯЦИОННЫХ ФУНКЦИЙ ОДНОМЕРНЫХ БОЗОНОВ}

\begin{abstract}
Обсуждаются различные асимптотические представления для корреляционных функций в критических интегрируемых системах. На примере модели одномерных бозонов доказано, что асимптотический ряд для корреляционных функций, полученный методом кратных интегралов, в пределе низкой температуры совпадает с предсказаниями конформной теории поля.
\end{abstract}

Ключевые слова: корреляционные функции, асимптотика.

\section{1. ВВЕДЕНИЕ}

Настоящая статья является кратким обзором результатов, полученных в рабо$\operatorname{Tax}[1]-[3]$.

Одной из наиболее важных и сложных проблем в теории интегрируемых систем является исследование корреляционных функций. В критических моделях поведение корреляционных функций при большом расстоянии описывается с помощью конформной теории поля (КТП) [4]-[6]. Допустим, что мы имеем некоторый оператор $\mathcal{O}(x)$, зависящий от пространственной переменной $x$. Тогда при достаточно малой температуре $T$ и при $x \rightarrow \infty$ асимптотическое поведение двухточечной функции дается формулой

$$
\langle\mathcal{O}(x) \mathcal{O}(0)\rangle_{T}=\sum_{\ell \in \mathbb{Z}} A_{\ell} e^{i k_{\ell} x}\left(\frac{\gamma T}{\operatorname{sh} \gamma T x}\right)^{\theta_{\ell}}, \quad x \rightarrow \infty .
$$

Для моделей, решаемых с помощью анзаца Бете [7]-[10], константы $\theta_{\ell}, k_{\ell}, \gamma$ можно найти из анализа поправок конечного объема [11]-[16]. Постоянные амплитуды $A_{\ell}$ в рамках данного метода остаются неопределенными. Отметим также, что представление (1.1) не является полным асимптотическим рядом. В нем приведены лишь главные члены разложения, соответствующие различным осциллирующим гармоникам $e^{i k_{\ell} x}$. Поправки к этим членам в настоящей статье не рассматриваются.

* Математический институт им. В. А. Стеклова РАН, Москва, Россия. E-mail: nslavnov@mi.ras.ru 
С другой стороны, для некоторых интегрируемых моделей (таких, как цепочки Гейзенберга, одномерные бозоны и др.) поведение корреляционных функций при конечной температуре и большом расстоянии может быть найдено с помощью метода квантовой трансфер-матрицы (KTM) [17]-[22]. В рамках данного метода асимптотическое разложение двухточечных функций имеет вид

$$
\langle\mathcal{O}(x) \mathcal{O}(0)\rangle_{T}=\sum_{i \geqslant 0} B_{i} e^{-x p_{i}}, \quad x \rightarrow \infty
$$

Здесь обратные корреляционные радиусы $p_{i}$ могут быть выражены через собственные значения $\Lambda_{i}$ KTM. Последние описываются в терминах решений нелинейных интегральных уравнений, которые называются уравнениями термодинамического анзаца Бете (ТАБ) [23]-[26]. Аналогично формуле (1.1) постоянные амплитуды $B_{i}$ остаются неопределенными, хотя метод КТМ позволяет связать их со скалярными произведениями, где один из векторов является собственным вектором КТМ.

Сравнивая разложения (1.1) и (1.2), мы видим, что они имеют различную структуру. Напомним, что метод КTM дает асимптотическое поведение корреляционных функций при любой конечной температуре, в то время как подход КТП работает только при достаточно малых $T$. Естественно ожидать, что при $T \rightarrow 0$ формула (1.2) переходит в (1.1). Однако если такой эффект действительно имеет место, то это означает, что предсказания КТП являются результатом пересуммирования ряда (1.2). Ясно, что такое пересуммирование невозможно осуществить явно до тех пор, пока постоянные амплитуды $B_{i}$ остаются неизвестными.

В настоящей статье мы изучаем проблему эквивалентности представлений (1.1) и (1.2) при низкой температуре. Для этого мы рассматриваем конкретный пример модели одномерных бозонов. Корреляционные функции в этой модели могут быть вычислены с помощью метода, основанного на представлении кратных интегралов. В частности, этот метод, примененный к температурным корреляционным функциям, дает асимптотическое разложение, аналогичное (1.2). Однако в отличие от подхода КТМ метод кратных интегралов позволяет получить явные представления для амплитуд $B_{i}$. Исследуя их поведение при $T \rightarrow 0$, мы можем пересуммировать ряд (1.2) в разложение (1.1).

Модель одномерных бозонов с двухчастичным дельтаобразным взаимодействием описывается квантовым нелинейным уравнением Шредингера. Гамильтониан этой модели равен

$$
H=\int_{0}^{L}\left(\partial_{x} \Psi^{\dagger} \partial_{x} \Psi+c \Psi^{\dagger} \Psi^{\dagger} \Psi \Psi-h \Psi^{\dagger} \Psi\right) d x .
$$

Здесь $\Psi$ и $\Psi^{\dagger}$ суть бозе-поля с каноническими одновременнь́ми коммутационными соотношениями, $c>0$ - константа связи и $h>0$ - химический потенциал.

В рамках алгебраического анзаца Бете [27]-[29] собственные функции $|\psi\rangle$ гамильтониана $H$ параметризуются наборами вещественных чисел:

$$
|\psi\rangle=\left|\psi\left(\lambda_{1}, \ldots, \lambda_{N}\right)\right\rangle, \quad N=0,1, \ldots,
$$

где $\lambda_{j}$ являются решениями системы уравнений Бете [7]. Мы будем исследовать корреляционные функции этой модели в термодинамическом пределе: $L, N \rightarrow \infty$ при фиксированной средней плотности $N / L=D$. 
Температурные корреляционные функции определяются стандартным образом:

$$
\langle\mathcal{O}(x) \mathcal{O}(0)\rangle_{T}=\frac{\operatorname{tr}\left(\mathcal{O}(x) \mathcal{O}(0) e^{-H / T}\right)}{\operatorname{tr}\left(e^{-H / T}\right)} .
$$

Как было показано в работах [30], [31], в модели одномерных бозонов данное определение приводит к следующей формуле:

$$
\langle\mathcal{O}(x) \mathcal{O}(0)\rangle_{T}=\lim _{N, L \rightarrow \infty} \frac{\left\langle\Omega_{T}|\mathcal{O}(x) \mathcal{O}(0)| \Omega_{T}\right\rangle}{\left\langle\Omega_{T} \mid \Omega_{T}\right\rangle},
$$

где $\left|\Omega_{T}\right\rangle$ - любое собственное состояние гамильтониана $H$, которое стремится к состоянию термодинамического равновесия в термодинамическом пределе.

Состояние термодинамического равновесия в модели одномерных бозонов описывается уравнением Янга-Янга [23] для энергии элементарных возбуждений $\varepsilon(\lambda)$,

$$
\varepsilon(\lambda)=\lambda^{2}-h-\frac{T}{2 \pi} \int_{\mathbb{R}} K(\lambda-\mu) \ln \left(1+e^{-\varepsilon(\mu) / T}\right) d \mu,
$$

и интегральным уравнением для полной плотности вакансий $\rho_{\mathrm{t}}(\lambda)$,

$$
\rho_{\mathrm{t}}(\lambda)-\frac{1}{2 \pi} \int_{\mathbb{R}} K(\lambda-\mu) \vartheta(\mu) \rho_{\mathrm{t}}(\mu) d \mu=\frac{1}{2 \pi} .
$$

Ядро $K(\lambda)$ и фермиевский вес $\vartheta(\lambda)$, возникающие в приведенных выше формулах, имеют вид

$$
K(\lambda)=\frac{2 c}{\lambda^{2}+c^{2}}, \quad \vartheta(\lambda)=\left(1+e^{\varepsilon(\lambda) / T}\right)^{-1} .
$$

Отметим, что уравнение (1.3) является типичным представителем уравнений ТАБ.

В дальнейшем мы рассмотрим конкретный пример производящей функции двухточечного коррелятора плотностей $j(x)=\Psi^{\dagger}(x) \Psi(x)$. Введем оператор числа частиц $\mathcal{Q}_{x}$ на интервале $[0, x]$ :

$$
\mathcal{Q}_{x}=\int_{0}^{x} j(z) d z
$$

Тогда производящая функция для двухточечного коррелятора плотностей имеет вид

$$
\left\langle e^{2 \pi i \alpha \mathcal{Q}_{x}}\right\rangle_{T}=\lim _{N, L \rightarrow \infty} \frac{\left\langle\Omega_{T}\left|e^{2 \pi i \alpha \mathcal{Q}_{x}}\right| \Omega_{T}\right\rangle}{\left\langle\Omega_{T} \mid \Omega_{T}\right\rangle},
$$

где $\alpha$ - некоторое комплексное число. Двухточечная корреляционная функция плотностей $\langle j(x) j(0)\rangle_{T}$ может быть получена из (1.6):

$$
\langle j(x) j(0)\rangle_{T}=-\left.\frac{1}{8 \pi^{2}} \frac{\partial^{2}}{\partial x^{2}} \frac{\partial^{2}}{\partial \alpha^{2}}\left\langle e^{2 \pi i \alpha \mathcal{Q}_{x}}\right\rangle_{T}\right|_{\alpha=0} .
$$

Мы ограничимся исследованием лишь производящей функции (1.6). Асимптотическое разложение для двухточечной функции $\langle j(x) j(0)\rangle_{T}$ можно найти в работах [1], [2].

Статья построена следующим образом. В разделе 2 приведено асимптотическое разложение для производящей функции (1.6), полученное методом кратных интегралов. Результаты записаны в терминах решений уравнений ТАБ. В разделе 3 рассмотрена термодинамика модели одномерных бозонов при низкой температуре. В разделе 4 дано решение уравнений ТАБ при низкой температуре. В разделе 5 доказана эквивалентность представлений (1.1) и (1.2) в пределе $T \rightarrow 0$. 


\section{2. АСИМПТОТИЧЕСКОЕ ПОВЕДЕНИЕ ПРОИЗВОДЯЩЕЙ ФУНКЦИИ}

Асимптотическое поведение производящей функции $\left\langle e^{2 \pi i \alpha \mathcal{Q}_{x}}\right\rangle_{T}$ дается в терминах решений нелинейных интегральных уравнений, аналогичных уравнению (1.3). Выберем $n$ точек $(n=0,1, \ldots) s_{j}^{+}$в верхней полуплоскости и $n$ точек $s_{j}^{-}$в нижней полуплоскости. Введем функцию $u(\lambda)$, удовлетворяющую нелинейному интегральному уравнению ТАБ:

$$
u(\lambda)=\lambda^{2}-h_{\alpha}-\frac{T}{2 \pi} \int_{\mathbb{R}} K(\lambda-\mu) \ln \left(1+e^{-u(\mu) / T}\right) d \mu+i T \sum_{j=1}^{n}\left(\theta\left(\lambda-s_{j}^{+}\right)-\theta\left(\lambda-s_{j}^{-}\right)\right),
$$

где $h_{\alpha}=h+2 \pi i \alpha T$ и

$$
\theta(\lambda)=i \ln \left(\frac{i c+\lambda}{i c-\lambda}\right), \quad \theta^{\prime}(\lambda)=K(\lambda)
$$

Ясно, что решение уравнения (2.1) зависит от параметров $\left.\left\{s^{ \pm}\right\}: u(\lambda)=u\left(\lambda \mid\left\{s^{ \pm}\right\}\right\}\right)$. Наложим условия

$$
1+\exp \left(-\frac{\left.u\left(s_{j}^{ \pm} \mid\left\{s^{ \pm}\right\}\right\}\right)}{T}\right)=0, \quad j=1, \ldots, n .
$$

Решения $\left\{s^{ \pm}\right\}_{i}$ данной системы уравнений фиксируют функции $u_{i}(\lambda)=u_{i}\left(\lambda \mid\left\{s^{ \pm}\right\}_{i}\right)$, удовлетворяющие интегральному уравнению (2.1) с дополнительным условием (2.2). Индекс $i$ нумерует различные наборы корней и соответствующие функции $u_{i}(\lambda)$. Отметим, что числа $\left\{s^{ \pm}\right\}_{i}$ не обязательно являются единственными нулями функции $1+e^{-u_{i}(\lambda) / T}$. Данная функция может обращаться в ноль и при некоторых значениях $\lambda=w_{p}, p=1,2, \ldots$, таких, что $w_{p} \notin\left\{s^{ \pm}\right\}_{i}$. Эти корни называются решениями типа дырки.

Удобно ввести вспомогательные функции $z_{i}(\lambda)$ :

$$
z_{i}(\lambda)=-\frac{1}{2 \pi} \ln \left(\frac{1+e^{-u_{i}(\lambda) / T}}{1+e^{-\varepsilon(\lambda) / T}}\right) .
$$

Тогда асимптотическое разложение производящей функции $\left\langle e^{2 \pi i \alpha \mathcal{Q}_{x}}\right\rangle_{T}$ при большом расстоянии может быть записано в виде суммы по функциям $z_{i}(\lambda)$ :

$$
\left\langle e^{2 \pi i \alpha \mathcal{Q}_{x}}\right\rangle_{T}=\sum_{i} e^{-x p\left[z_{i}\right]} B\left[z_{i}\right]+o\left(e^{-x \max \operatorname{Re}\left(p\left[z_{i}\right]\right)}\right)
$$

Здесь $p\left[z_{i}\right]$ и $B\left[z_{i}\right]$ являются некоторыми функционалами от $z_{i}(\lambda)$. Их явные представления будут даны ниже.

Уравнение (2.1) может быть записано в виде уравнения Янга-Янга с деформированным контуром. Для этого мы прежде всего определим полюсы фермиевского веса $\vartheta(\lambda)(1.5)$. Пусть числа $\left\{r_{j}^{ \pm}\right\}$являются корнями уравнения $1+e^{-\varepsilon(r) / T}=0$, причем $r_{j}^{+}$принадлежат верхней полуплоскости, а $r_{j}^{-}-$нижней (см. рис. 1$)$. Тогда функция $u_{i}(\lambda)$ удовлетворяет уравнению

$$
u_{i}(\lambda)=\lambda^{2}-h_{\alpha}-\frac{T}{2 \pi} \int_{\mathcal{C}_{i}} K(\lambda-\mu) \ln \left(1+e^{-u_{i}(\mu) / T}\right) d \mu .
$$




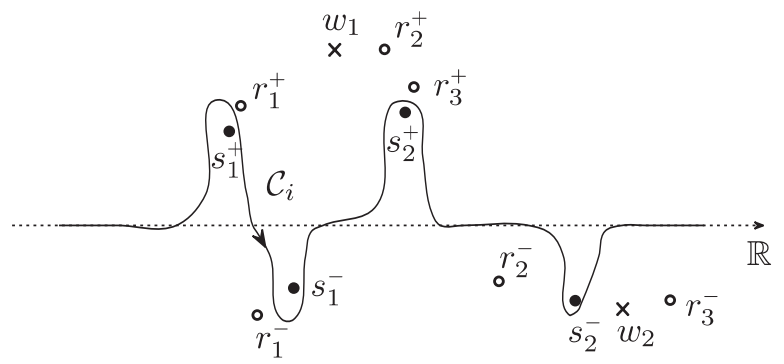

Рис. 1. Контур интегрирования $\mathcal{C}_{i}$. Полюсы фермиевского веса $r^{ \pm}$обозначены как $\circ$, нули $s^{ \pm}-$как $\bullet$, решения типа дырок $w$ обозначены как $\times$. Контур $\mathcal{C}_{i}$ является деформацией вещественной оси, которая не пересекает полюсы фермиевского веса $r^{ \pm}$и решения типа дырок $w$. При этом точки $s_{1}^{+}, s_{2}^{+}$оказываются справа от контура, а точки $s_{1}^{-}, s_{2}^{-}-$ слева.

Здесь контур интегрирования $\mathcal{C}_{i}$ является деформацией вещественной оси такой, что при этой деформации $\mathcal{C}_{i}$ пересекает только корни $\left\{s^{ \pm}\right\}_{i}$. При этом полюсы $\left\{r_{j}^{ \pm}\right\}$ фермиевского веса и решения типа дырок контур не пересекает (см. рис. 1). Тогда асимптотическое разложение производящей функции $\left\langle e^{2 \pi i \alpha \mathcal{Q}_{x}}\right\rangle_{T}$ может быть интерпретировано как сумма по всем возможным неэквивалентным выборам контура $\mathcal{C}_{i}$. Такое представление для корреляционных функций в точности соответствует их описанию на языке метода КTM.

Явные формулы для показателей экспоненциального убывания $p\left[z_{i}\right]$ и амплитуд $B\left[z_{i}\right]$ могут быть записаны в виде интегралов по контурам $\mathcal{C}_{i}$. В частности, обратные корреляционные радиусы $p\left[z_{i}\right]$ имеют очень простой вид:

$$
p\left[z_{i}\right]=\int_{\mathcal{C}_{i}} z_{i}(\lambda) d \lambda .
$$

Выражения для амплитуд $B\left[z_{i}\right]$ гораздо более громоздки. Мы приводим здесь лишь ту часть амплитуды, которая нетривиально зависит от температуры при $T \rightarrow 0$ :

$$
B\left[z_{i}\right]=\widetilde{B}\left[z_{i}\right] B_{\mathrm{s}}\left[z_{i}\right], \quad B_{\mathrm{s}}\left[z_{i}\right]=\exp \left(-\int_{\mathcal{C}_{i}} \frac{z_{i}(\lambda) z_{i}(\mu)}{\left(\lambda-\mu_{+}\right)^{2}} d \lambda d \mu\right),
$$

где символ $\mu_{+}$означает, что переменная $\mu$ слегка сдвинута влево от контура интегрирования $\mathcal{C}_{i}$. Функционал $\widetilde{B}\left[z_{i}\right]$ имеет конечный предел при $T=0$. Явные формулы для него можно найти в работах [1], [2].

Таким образом, мы описали асимптотическое поведение производящей функции (1.6). Оно дается в терминах решений уравнений ТАБ. В следующих разделах мы исследуем эти уравнения при малых $T$.

\section{3. ТЕРМОДИНАМИКА ПРИ НИЗКОЙ ТЕМПЕРАТУРЕ}

В этом разделе мы приводим список формул, описывающих термодинамику модели одномерных бозонов при низкой температуре. Рассмотрим сначала случай $T=0$. 
Известно [23], что решение $\varepsilon(\lambda)$ уравнения (1.3) имеет два корня $\pm q(T)$ на вещественной оси: $\varepsilon( \pm q(T))=0$. При этом $\varepsilon(\lambda)>0$ для $|\lambda|>q(T)$ и $\varepsilon(\lambda)<0$ для $|\lambda|<q(T)$. Пусть $\varepsilon(\lambda) \rightarrow \varepsilon_{0}(\lambda)$ и $q(T) \rightarrow q$ при $T \rightarrow 0$. Тогда

$$
\lim _{T \rightarrow 0} T \ln \left(1+e^{-\varepsilon(\lambda) / T}\right)= \begin{cases}0, & |\lambda|>q, \\ -\varepsilon_{0}(\lambda), & |\lambda|<q .\end{cases}
$$

Легко видеть, что в этом случае уравнение (1.3) превращается в линейное интегральное уравнение для функции $\varepsilon_{0}(\lambda)$ :

$$
\varepsilon_{0}(\lambda)-\frac{1}{2 \pi} \int_{-q}^{q} K(\lambda-\mu) \varepsilon_{0}(\mu) d \mu=\lambda^{2}-h, \quad \varepsilon_{0}( \pm q)=0 .
$$

Решение уравнения (3.1) имеет смысл одетой энергии возбуждений в основном состоянии [9], [10]. Интервал $[-q, q]$ называется зоной Ферми.

При $T=0$ состояние термодинамического равновесия стремится к основному состоянию модели одномерных бозонов. Легко видеть, что фермиевский вес $\vartheta(\lambda)(1.5)$ превращается в характеристическую функцию интервала $[-q, q]$, поэтому уравнение (1.4) для плотности принимает вид

$$
\rho_{\mathrm{t}}(\lambda)-\frac{1}{2 \pi} \int_{-q}^{q} K(\lambda-\mu) \rho_{\mathrm{t}}(\mu) d \mu=\frac{1}{2 \pi}, \quad T=0 .
$$

Еще одной важной характеристикой основного состояния является одетый заряд $Z(\lambda)$. В модели одномерных бозонов он пропорционален плотности: $Z(\lambda)=2 \pi \rho_{\mathrm{t}}(\lambda)$. Ниже мы будем использовать специальное обозначение $\mathcal{Z}=Z( \pm q)$ для величины одетого заряда на границе зоны Ферми. Как следует из уравнения (3.2), формальное выражение для $\mathcal{Z}$ дается в терминах резольвенты оператора $I-\frac{1}{2 \pi} K$, действующего на интервале $[-q, q]$ :

$$
\mathcal{Z}=1+\int_{-q}^{q} R(\lambda, \pm q) d \lambda
$$

где

$$
R(\lambda, \xi)-\frac{1}{2 \pi} \int_{-q}^{q} K(\lambda-\mu) R(\mu, \xi) d \mu=\frac{1}{2 \pi} K(\lambda-\xi)
$$

Приведем также формулы для некоторых констант, которые встретятся в дальнейшем, а именно для импульса Ферми $k_{\mathrm{F}}$ и скорости звука $v_{0}$ на границе зоны Ферми:

$$
k_{\mathrm{F}}=\pi \int_{-q}^{q} \rho_{\mathrm{t}}(\lambda) d \lambda, \quad v_{0}=\frac{\varepsilon_{0}^{\prime}}{\mathcal{Z}},
$$

где мы обозначили $\varepsilon_{0}^{\prime} \equiv \varepsilon_{0}^{\prime}(q)$.

При низкой, но ненулевой температуре в уравнении Янга-Янга возникают степенные поправки по $T$. Обсудим вкратце их происхождение. 
Интеграл в уравнении (1.3) берется по всей вещественной оси. Рассмотрим область интегрирования вне зоны Ферми, где $\varepsilon(\mu)>0$. Ясно, что при малых $T$ функция $\ln \left(1+e^{-\varepsilon(\mu) / T}\right)$ равномерно экспоненциально мала, если $\mu$ находится на любом конечном расстоянии от границ зоны Ферми $\pm q$. Поэтому вклады в интеграл от этих областей имеют порядок $O\left(T^{\infty}\right)$. Внутри зоны Ферми, где $\varepsilon(\mu)<0$, мы имеем

$$
T \ln \left(1+e^{-\varepsilon(\mu) / T}\right)=-\varepsilon(\mu)+T \ln \left(1+e^{\varepsilon(\mu) / T}\right),
$$

и теперь уже второе слагаемое в правой части дает экспоненциально малые вклады в уравнение. Таким образом, единственными областями интегрирования, которые могут дать степенные вклады по $T$, оказываются $\delta$-окрестности точек $\pm q$ такие, что $\delta \rightarrow 0$ при $T \rightarrow 0$. В этих окрестностях функция $\varepsilon(\mu)$ может быть заменена первым неисчезающим членом своего разложения в ряд Тейлора. После этого вклады от $\delta$-окрестностей границ зоны Ферми $\pm q$ вычисляются явно (см. приложение).

Приведенные выше рассуждения позволяют искать решение уравнения (1.3) в виде степенного ряда по T [2], [32]-[34]:

$$
\varepsilon(\lambda)=\sum_{k=0}^{\infty} T^{k} \varepsilon_{k}(\lambda) .
$$

При этом функция $\varepsilon_{0}(\lambda)$ предполагается заданной. В рамках такого подхода интегральные уравнения для всех функций $\varepsilon_{k}(\lambda)$ становятся линейными благодаря свойству (3.5). Их решения выражаются через резольвенту (3.3). Для наших целей достаточно найти линейные по $T$ поправки к функциям $z_{i}(\lambda)$. При этом следует учитывать, что функции $u_{i}(\lambda)$ и $\varepsilon(\lambda)$ входят в определение $(2.3)$ с коэффициентом $1 / T$, поэтому для нахождения линейных поправок к $z_{i}(\lambda)$ следует найти $u_{i}(\lambda)$ и $\varepsilon(\lambda)$ с точностью до второго порядка по $T$. Подробное вычисление поправок $\varepsilon_{k}(\lambda)$ дано в работе [2]. Оказывается, что $\varepsilon_{1}(\lambda)=0$ и

$$
\varepsilon_{2}(\lambda)=-\frac{\pi^{2}}{6 \varepsilon_{0}^{\prime}}(R(\lambda, q)+R(\lambda,-q)),
$$

где $R(\lambda, \mu)$ - резольвента, определенная формулой (3.3).

В дальнейшем при выборе контуров интегрирования $\mathcal{C}_{i}$ нам потребуется также знать положение корней фермиевского веса $r_{k}^{ \pm}$при малой температуре. Для этого нам следует решить уравнение

$$
\varepsilon(\lambda)=2 \pi i T\left(k+\frac{1}{2}\right), \quad k \in \mathbb{Z},
$$

в линейном приближении по $T$. Очевидно, что при $T \rightarrow 0$ все корни стремятся к $q$ или $-q$ (см. рис. 2$)$. Разлагая $\varepsilon(\lambda)$ в ряд в окрестностях точек $\pm q$, находим с учетом равенства $\varepsilon( \pm q)=0$, что

$$
\begin{aligned}
& r_{k}^{+}= \pm q+\frac{2 \pi i T}{\varepsilon_{0}^{\prime}}\left(k+\frac{1}{2}\right)+O\left(T^{2}\right), \quad k \geqslant 0, \\
& r_{k}^{-}= \pm q+\frac{2 \pi i T}{\varepsilon_{0}^{\prime}}\left(k+\frac{1}{2}\right)+O\left(T^{2}\right), \quad k<0 .
\end{aligned}
$$

Таким образом, в линейном приближении по $T$ мы получаем две серии корней. Мы 


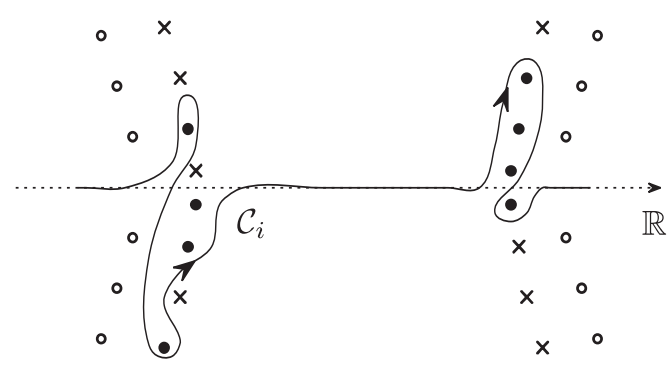

Рис. 2. При $T \rightarrow 0$ полюсы фермиевского веса $r^{ \pm}$(обозначенные как ), нули $s^{ \pm}$( ) и решения типа дырок $w($ ) стремятся к границам зоны Ферми $\pm q$.

будем называть корни, сходящиеся к $-q$, левой серией, а корни, сходящиеся к $q,-$ правой серией.

\section{4. РЕШЕНИЕ ИНТЕГРАЛЬНОГО УРАВНЕНИЯ В НИЗКОТЕМПЕРАТУРНОМ ПРЕДЕЛЕ}

Зафиксируем некоторый контур $\mathcal{C}_{i}$ и рассмотрим соответствующий вклад в асимптотику производящей функции. Пусть контур $\mathcal{C}_{i}$ обходит $n$ точек $s^{+}$в верхней полуплоскости сверху и $n$ точек $s^{-}$в нижней полуплоскости снизу, где $n$ - произвольное неотрицательное целое число. Важно зафиксировать предельные значения выбранных корней $s^{ \pm}$при $T=0$.

В пределе $T \rightarrow 0$ уравнение $(2.1)$ совпадает с пределом $T \rightarrow 0$ уравнения ЯнгаЯнга, следовательно, $\left.u_{i}(\lambda)\right|_{T=0}=\varepsilon_{0}(\lambda)$. Естественно ожидать, что аналогично точкам $r_{k}^{ \pm}$корни $s_{k}^{ \pm}$сходятся к $q$ или $-q$ при $T \rightarrow 0$ (см. рис. 2). Таким образом, в низкотемпературном пределе эти корни должны образовать две серии. Пусть $n_{\mathrm{p}}^{+}$ корней $s^{+}$и $n_{\mathrm{h}}^{+}$корней $s^{-}$принадлежат правой серии и соответственно $n_{\mathrm{p}}^{-}$корней $s^{+}$ и $n_{\mathrm{h}}^{-}$корней $s^{-}$принадлежат левой серии. Очевидно, что введенные целые числа удовлетворяют соотношениям

$$
n_{\mathrm{p}}^{+}+n_{\mathrm{p}}^{-}=n_{\mathrm{h}}^{+}+n_{\mathrm{h}}^{-}=n, \quad n_{\mathrm{p}}^{+}-n_{\mathrm{h}}^{+}=n_{\mathrm{h}}^{-}-n_{\mathrm{p}}^{-}=\ell,
$$

где $\ell$ - фиксированное целое число из интервала $-n \leqslant \ell \leqslant n$.

Опишем теперь процедуру построения решения уравнения (2.1) при малых $T$. Аналогично рассмотренному выше случаю уравнения (1.3) мы ищем решение в виде степенного ряда по $T$. При этом нам достаточно ограничиться линейными и квадратичными поправками, т. е.

$$
u_{i}(\lambda)=\sum_{k=0}^{2} T^{k} u_{i ; k}(\lambda)+O\left(T^{3}\right),
$$

где, как уже отмечалось,$u_{i ; 0}(\lambda)=\varepsilon_{0}(\lambda)$, а все корни $s^{ \pm}$совпадают с $q$ или $-q$ при $T=0$. Степенные поправки к $u_{i ; 0}(\lambda)$ вычисляются тем же методом, что и поправки к $\varepsilon_{0}(\lambda)$. Оказывается, что линейная поправка $u_{i ; 1}(\lambda)$ зависит не от конкретных значений корней $s^{ \pm}$, а лишь от их распределения между правой и левой серией:

$$
u_{i ; 1}(\lambda)=-2 \pi i \alpha_{\ell} Z(\lambda)+2 \pi i \ell, \quad \alpha_{\ell}=\alpha+\ell,
$$


где $Z(\lambda)$ - одетый заряд, а $\ell$ введено в (4.1). Легко проверить, что при этом мы имеем

$$
\left.z_{i}(\lambda)\right|_{T=0}=-\frac{u_{i ; 1}(\lambda)}{2 \pi} \chi_{[-q, q]}(\lambda)
$$

где $\chi_{[-q, q]}(\lambda)$ - характеристическая функция интервала $[-q, q]$.

Полученный результат позволяет вычислить линейные поправки к корням $s^{ \pm}$. Пусть

$$
\begin{array}{ll}
\left\{s^{+}\right\}=\left\{q+i T \eta^{+}\right\}_{n_{\mathrm{p}}^{+}} \bigcup\left\{-q+i T \eta^{-}\right\}_{n_{\mathrm{p}}^{-}}+O\left(T^{2}\right), & \operatorname{Re}\left(\eta^{ \pm}\right)>0 \\
\left\{s^{-}\right\}=\left\{q-i T \xi^{+}\right\}_{n_{\mathrm{h}}^{+}} \bigcup\left\{-q-i T \xi^{-}\right\}_{n_{\mathrm{h}}^{-}}+O\left(T^{2}\right), & \operatorname{Re}\left(\xi^{ \pm}\right)>0
\end{array}
$$

Тогда

$$
\begin{aligned}
& \varepsilon_{0}^{\prime} \eta_{k}^{ \pm}=2 \pi\left(p_{k}^{ \pm}-\frac{1}{2}\right) \pm i u_{i ; 1}(q), \\
& \varepsilon_{0}^{\prime} \xi_{k}^{ \pm}=2 \pi\left(h_{k}^{ \pm}-\frac{1}{2}\right) \mp i u_{i ; 1}(q),
\end{aligned}
$$

где $p_{k}^{ \pm}$и $h_{k}^{ \pm}$суть некоторые положительные целые числа. Выбор конкретного набора попарно различных целых чисел ${ }^{1)}$ задает конкретную функцию $u_{i}(\lambda)$. В этом смысле индекс $i$ у функций $u_{i}(\lambda)$ является мульти-индексом, который, помимо чисел $p_{k}^{ \pm}$ и $h_{k}^{ \pm}$, включает в себя также целые числа $n$ и $\ell$.

Зная линейные поправки к функции $u_{i}(\lambda)$ и к корням $s^{ \pm}$, мы можем найти $u_{i ; 2}(\lambda)$ :

$$
\begin{aligned}
u_{i ; 2}(\lambda)= & R(\lambda, q)\left[2 \pi \sum_{j=1}^{n_{\mathrm{p}}^{+}} \eta_{j}^{+}+2 \pi \sum_{j=1}^{n_{\mathrm{h}}^{+}} \xi_{j}^{+}-\frac{1}{2 \varepsilon_{0}^{\prime}}\left(\frac{\pi^{2}}{3}+u_{i ; 1}^{2}(q)\right)\right]+ \\
& +R(\lambda,-q)\left[2 \pi \sum_{j=1}^{n_{\mathrm{p}}^{-}} \eta_{j}^{-}+2 \pi \sum_{j=1}^{n_{\mathrm{h}}^{-}} \xi_{j}^{-}-\frac{1}{2 \varepsilon_{0}^{\prime}}\left(\frac{\pi^{2}}{3}+u_{i ; 1}^{2}(q)\right)\right]
\end{aligned}
$$

где $R(\lambda, \mu)$ - резольвента оператора $I-\frac{1}{2 \pi} K(3.3)$.

Подставляя выражения для $u_{i ; k}(\lambda)$ в формулы $(2.3),(2.5)$, мы после несложных вычислений находим

$p\left[z_{i}\right]=-2 i \alpha_{\ell} k_{\mathrm{F}}+\frac{2 \pi T}{v_{0}}\left[\left(\alpha_{\ell} \mathcal{Z}\right)^{2}-\ell^{2}-n+\sum_{j=1}^{n_{\mathrm{p}}^{+}} p_{j}^{+}+\sum_{j=1}^{n_{\mathrm{p}}^{-}} p_{j}^{-}+\sum_{j=1}^{n_{\mathrm{h}}^{+}} h_{j}^{+}+\sum_{j=1}^{n_{\mathrm{h}}^{-}} h_{j}^{-}\right]+O\left(T^{2}\right)$.

Напомним, что $\mathcal{Z}=Z(q), k_{\mathrm{F}}$ - импульс Ферми, $v_{0}$ - скорость звука на границе зоны Ферми (см. формулы (3.4)).

Предельное значение амплитуды $B\left[z_{i}\right]$ состоит из двух частей. Как мы уже отмечали, множитель $\widetilde{B}\left[z_{i}\right]$ имеет конечный предел при $T=0$. В силу условия (4.4) он превращается в константу $\widetilde{B}_{\ell}$, зависящую от целого числа $\ell$. Формально мы должны учесть также поправки к этому функционалу, линейные по T. Можно показать,

1) Мы имеем в виду, что $p_{j}^{+} \neq p_{k}^{+}, p_{j}^{-} \neq p_{k}^{-}$и аналогично для чисел $h^{ \pm}$. При этом возможно, что $p_{j}^{+}=p_{k}^{-}, p_{j}^{+}=h_{k}^{+}$и т. п. 
однако, что учет старших порядков по $T$ приводит к появлению поправочных слагаемых в асимптотическом разложении производящей функции. Как уже отмечалось во введении, такими поправками мы пренебрегаем.

Вычисление низкотемпературного поведения функционала $B_{\mathrm{s}}\left[z_{i}\right]$ производится явно. Особенность двойного интеграла

$$
J\left[z_{i}\right]=-\int_{\mathcal{C}_{i}} \frac{z_{i}(\lambda) z_{i}(\mu)}{\left(\lambda-\mu_{+}\right)^{2}} d \lambda d \mu
$$

состоит в том, что он хорошо определен при любом $T>0$, но не имеет конечного предела при $T=0$. Действительно, если формально подставить (4.4) в (4.5), то возникающий при этом интеграл расходится. Воспользовавшись формулой (4.3), несложно убедиться в том, что при малых $T$ интеграл $J\left[z_{i}\right]$ ведет себя как

$$
J\left[z_{i}\right]=-2 \alpha_{\ell}^{2} \mathcal{Z}^{2} \ln T+O(1), \quad T \rightarrow 0 .
$$

Сложнее найти конечный вклад $O(1)$ в этот интеграл. Мы отсылаем читателя за подробным анализом интеграла (4.5) к работе [2] и приводим окончательный ответ:

$$
\begin{aligned}
B_{\mathrm{s}}^{(0)}\left[z_{i}\right]= & \lim _{T \rightarrow 0} B_{\mathrm{s}}\left[z_{i}\right]\left|\frac{q \varepsilon_{0}^{\prime}}{i \pi T}\right|^{2 \alpha_{\ell}^{2} \mathcal{Z}^{2}}=e^{\alpha_{\ell}^{2} C_{1}[Z]}\left(\frac{\sin \pi \alpha_{\ell} \mathcal{Z}}{\pi}\right)^{2 n} G^{2}\left(1, \alpha_{\ell} \mathcal{Z}-\ell\right) \times \\
& \times R_{n_{\mathrm{p}}^{+}, n_{\mathrm{h}}^{+}}\left(\left\{p^{+}\right\},\left\{h^{+}\right\} \mid \alpha_{\ell} \mathcal{Z}\right) R_{n_{\mathrm{p}}^{-}, n_{\mathrm{h}}^{-}}\left(\left\{p^{-}\right\},\left\{h^{-}\right\} \mid-\alpha_{\ell} \mathcal{Z}\right),
\end{aligned}
$$

где

$$
R_{n, m}(\{p\},\{h\} \mid F)=\frac{\prod_{j>k}^{n}\left(p_{j}-p_{k}\right)^{2} \prod_{j>k}^{m}\left(h_{j}-h_{k}\right)^{2}}{\prod_{j=1}^{n} \prod_{k=1}^{m}\left(p_{j}+h_{k}-1\right)^{2}} \prod_{k=1}^{n} \frac{\Gamma^{2}\left(p_{k}+F\right)}{\Gamma^{2}\left(p_{k}\right)} \prod_{k=1}^{m} \frac{\Gamma^{2}\left(h_{k}-F\right)}{\Gamma^{2}\left(h_{k}\right)} .
$$

Функция $G(x)$ в формуле (4.6) является функцией Барнса, а функционал $C_{1}[Z]$ имеет вид

$$
C_{1}[Z]=\frac{1}{2} \int_{-q}^{q} \frac{Z^{\prime}(\lambda) Z(\mu)-Z(\lambda) Z^{\prime}(\mu)}{\lambda-\mu} d \lambda d \mu+2 \mathcal{Z} \int_{-q}^{q} \frac{Z(\lambda)-\mathcal{Z}}{\lambda-q} d \lambda .
$$

Таким образом, мы нашли функционалы $p\left[z_{i}\right]$ и $B\left[z_{i}\right]$ в линейном приближении по $T$. Они параметризуются набором целых чисел $n \geqslant 0, \ell \in \mathbb{Z},\left\{p^{ \pm}>0\right\}$ и $\left\{h^{ \pm}>0\right\}$. Суммирование по функциям $z_{i}$ (или, что то же самое, суммирование по контурам $\mathcal{C}_{i}$ в формуле (2.4)) означает суммирование по вышеперечисленным целым числам.

\section{5. СУММИРОВАНИЕ АСИМПТОТИЧЕСКОГО РАЗЛОЖКНИЯ}

Полученные оценки для обратных корреляционных радиусов и амплитуд позволяют в низкотемпературном приближении переписать асимптотическое разложение (2.4) для производящей функции $\left\langle e^{2 i \pi \alpha \mathcal{Q}_{x}}\right\rangle_{T}$ в виде

$$
\left\langle e^{2 i \pi \alpha \mathcal{Q}_{x}}\right\rangle_{T} \simeq \sum_{\ell \in \mathbb{Z}} Q_{\ell} e^{2 i \alpha_{\ell} k_{\mathrm{F}} x}, \quad x \rightarrow \infty
$$


где коэффициенты $Q_{\ell}$ равны

$$
\begin{aligned}
Q_{\ell}= & \widetilde{B}_{\ell}\left(\frac{\pi T}{q \varepsilon_{0}^{\prime}}\right)^{2 \alpha_{\ell}^{2} \mathcal{Z}^{2}} G^{2}\left(1, \alpha_{\ell} \mathcal{Z}-\ell\right) \times \\
& \times \exp \left(\alpha_{\ell}^{2} C_{1}[Z]-\frac{2 \pi T x}{v_{0}}\left(\left(\alpha_{\ell} \mathcal{Z}\right)^{2}-\ell^{2}\right)\right) W_{+} W_{-} .
\end{aligned}
$$

Для того чтобы определить множители $W_{ \pm}$, мы прежде всего зададим функцию $W(\nu, r)$, зависящую от комплексного $\nu$ и целого $r$,

$$
\begin{aligned}
W(\nu, r)= & \sum_{\substack{n, n^{\prime} \geqslant 0, n-n^{\prime}=r}} \sum_{\substack{p_{n}>\cdots>p_{1} \geqslant 1, h_{n^{\prime}}>\cdots>h_{1} \geqslant 1}} \prod_{j=1}^{n} e^{-\left(2 \pi T x / v_{0}\right)\left(p_{j}-1\right)} \times \\
& \times \prod_{k=1}^{n^{\prime}} e^{-\left(2 \pi T x / v_{0}\right) h_{k}}\left(\frac{\sin \pi \nu}{\pi}\right)^{2 n^{\prime}} R_{n, n^{\prime}}(\{p\},\{h\} \mid \nu),
\end{aligned}
$$

где $R_{n, n^{\prime}}(\{p\},\{h\} \mid \nu)$ дается формулой (4.7). Тогда

$$
W_{ \pm}=\left.W(\nu, r)\right|_{\substack{\nu= \pm\left(\alpha_{\ell} \mathcal{Z}-\ell\right) \\ r= \pm \ell}} .
$$

Легко видеть, что множители $W_{ \pm}$соответствуют суммам по всем наборам целых чисел $\left\{p^{ \pm}\right\}$и $\left\{h^{ \pm}\right\}$, которые параметризуют возможные конфигурации корней $\left\{s^{ \pm}\right\}_{i}$ при фиксированном $\ell$. Такие суммы были вычислены в работах [35]-[38], [3]:

$$
W(\nu, r)=\frac{G^{2}(1+r+\nu)}{G^{2}(1+\nu)} \frac{e^{-\left(\pi T x / v_{0}\right) r(r-1)}}{\left(1-e^{-2 \pi T x / v_{0}}\right)^{(\nu+r)^{2}}} .
$$

Полагая $\nu= \pm\left(\alpha_{\ell} \mathcal{Z}-\ell\right), r= \pm \ell$ и подставляя (5.2) в (5.1), мы после несложных преобразований получаем

$$
Q_{\ell}=A_{\ell}\left(\frac{\pi T / v_{0}}{\operatorname{sh}\left(\pi T x / v_{0}\right)}\right)^{2 \alpha_{\ell}^{2} \mathcal{Z}^{2}}, \quad \text { где } \quad A_{\ell}=\widetilde{B}_{\ell} \frac{G^{2}\left(1, \alpha_{\ell} \mathcal{Z}\right)}{(2 q \mathcal{Z})^{2 \alpha_{\ell}^{2} \mathcal{Z}^{2}}} e^{\alpha_{\ell}^{2} C_{1}[Z]}
$$

и мы воспользовались тем, что $\varepsilon_{0}^{\prime}=\mathcal{Z} v_{0}$. Таким образом, мы в точности воспроизводим асимптотическое разложение (1.1):

$$
\left\langle e^{2 i \pi \alpha \mathcal{Q}_{x}}\right\rangle_{T} \simeq \sum_{\ell \in \mathbb{Z}} A_{\ell} e^{2 i \alpha_{\ell} k_{\mathrm{F}} x}\left(\frac{\pi T / v_{0}}{\operatorname{sh}\left(\pi T x / v_{0}\right)}\right)^{2 \alpha_{\ell}^{2} \mathcal{Z}^{2}}
$$

Мы приходим к выводу, что асимптотическое разложение (1.2) в низкотемпературном пределе становится эквивалентным разложению (1.1).

В заключение скажем несколько слов об амплитудах $A_{\ell}$ в формуле (5.3). Они зависят от констант $\widetilde{B}_{\ell}$, явный, но громоздкий вид которых можно найти в работах [1], [2]. В то же время эти амплитуды имеют простой физический смысл [39], [40].

Вернемся на время к модели одномерных бозонов на конечном интервале $L$. Основное состояние $\left|\psi_{0}\right\rangle$ этой модели в секторе с фиксированным числом частиц $N$ 
отвечает некоторому конкретному решению $\left|\psi_{0}\right\rangle=\left|\psi_{0}\left(\lambda_{1}, \ldots, \lambda_{N}\right)\right\rangle$ уравнений Бете. Положим $\tilde{\lambda}_{k}=\lambda_{k}+\alpha_{\ell} / L$ и введем вектор $\left|\tilde{\psi}_{0}\right\rangle=\left|\psi_{0}\left(\tilde{\lambda}_{1}, \ldots, \tilde{\lambda}_{N}\right)\right\rangle$. Определим нормированное скалярное произведение

$$
\mathcal{F}\left(\alpha_{\ell}\right)=\frac{\left\langle\tilde{\psi}_{0} \mid \psi_{0}\right\rangle}{\left\|\tilde{\psi}_{0}\right\| \cdot\left\|\psi_{0}\right\|} .
$$

Можно показать [39], что при большом $L$ величина $\mathcal{F}\left(\alpha_{\ell}\right)$ ведет себя как $L^{-2 \alpha_{\ell}^{2} \mathcal{Z}^{2}}$. Однако конечная часть $\mathcal{F}\left(\alpha_{\ell}\right)$ с точностью до числового коэффициента совпадает с амплитудой $A_{\ell}$, а именно

$$
A_{\ell}=\lim _{L, N \rightarrow \infty}\left(\frac{L}{2 \pi}\right)^{2 \alpha_{\ell}^{2} \mathcal{Z}^{2}}\left|\mathcal{F}\left(\alpha_{\ell}\right)\right|^{2} .
$$

В работах [39], [41] было установлено, что с точностью до фазового множителя скалярное произведение $\mathcal{F}\left(\alpha_{\ell}\right)$ равно матричному элементу оператора $e^{2 i \pi \alpha \mathcal{Q}_{x}}$ между состояниями $\left\langle\tilde{\psi}_{0}\right|$ и $\left|\psi_{0}\right\rangle$. Поэтому постоянные амплитуды $A_{\ell}$ в формуле (5.3) имеют смысл термодинамического предела матричных элементов оператора $e^{2 i \pi \alpha \mathcal{Q}_{x}}$, нормированных соответствующим образом.

\section{ПРИЛОЖЕНИЕ}

\section{Вычисление степенных поправок}

Рассмотрим интеграл вида

$$
J[\varepsilon]=T \int_{\mathbb{R}} f(\lambda) \ln \left(1+e^{-\varepsilon(\lambda) / T}\right) d \lambda,
$$

где функция $f(\lambda)$ ограничена на вещественной оси и дифференцируема в окрестностях точек $\pm q$. Как уже объяснялось в разделе 3 , в силу свойств функции $\varepsilon(\lambda)$ мы имеем

$$
\lim _{T \rightarrow 0} J[\varepsilon]=-\int_{-q}^{q} f(\lambda) \varepsilon_{0}(\lambda) d \lambda .
$$

Наша задача - найти степенные поправки к этому выражению при малых $T$. При этом мы будем искать функцию $\varepsilon(\lambda)$ в виде ряда $(3.6)$, в котором $\varepsilon_{0}(\lambda)$ считается известной функцией.

Пусть $\delta>0$ такова, что $\delta \rightarrow 0$ при $T \rightarrow 0$, в то время как $\delta / T \rightarrow \infty$ при $T \rightarrow 0$. Разобьем вещественную ось на пять областей:

$$
|\lambda|>q+\delta, \quad|\lambda|<q-\delta, \quad|\lambda \pm q|<\delta .
$$

Как уже объяснялось, интегралы по областям $|\lambda|>q+\delta$ дают вклады порядка $O\left(T^{\infty}\right)$. Пусть интеграл $J_{0}$ соответствует вкладу отрезка $|\lambda|<q-\delta$. В силу (3.5) имеем

$$
J_{0}[\varepsilon]=-\int_{-q+\delta}^{q-\delta} f(\lambda) \varepsilon(\lambda) d \lambda+O\left(T^{\infty}\right) .
$$

Наконец, интегралы $J_{ \pm q}$ соответствуют вкладам $\delta$-окрестностей точек $\pm q$. Именно эти интегралы дают степенные поправки при малых $T$. Рассмотрим $\delta$-окрестность 
точки $q$ и вычислим первую поправку к (П.1). В малой окрестности точки $q$ мы можем заменить $f(\lambda)$ на $f(q)$ и $\varepsilon(\lambda)$ - на $(\lambda-q) \varepsilon_{0}^{\prime}+T \varepsilon_{1}(q)$. Напомним, что $\varepsilon_{0}(q)=0$ и мы ввели обозначение $\varepsilon_{0}^{\prime} \equiv \varepsilon_{0}^{\prime}(q)$. Тогда

$$
J_{q}[\varepsilon]=T f(q) \int_{-\delta}^{\delta} \ln \left(1+e^{-\left(\lambda \varepsilon_{0}^{\prime} / T\right)-\varepsilon_{1}(q)}\right) d \lambda+\cdots,
$$

где многоточие означает поправки высших порядков по $T$. После замены переменных $\lambda=\mu T / \varepsilon_{0}^{\prime}$ мы получаем

$$
\begin{aligned}
J_{q}[\varepsilon]= & \frac{T^{2} f(q)}{\varepsilon_{0}^{\prime}} \int_{-\delta \varepsilon_{0}^{\prime} / T}^{\delta \varepsilon_{0}^{\prime} / T}\left[\ln \left(1+e^{-\mu-\varepsilon_{1}(q)}\right)+\left(\mu+\varepsilon_{1}(q)\right) \Theta\left(-\mu-\varepsilon_{1}(q)\right)\right] d \mu- \\
& -\frac{T^{2} f(q)}{\varepsilon_{0}^{\prime}} \int_{-\delta \varepsilon_{0}^{\prime} / T}^{-\varepsilon_{1}(q)}\left(\mu+\varepsilon_{1}(q)\right) d \mu+\cdots,
\end{aligned}
$$

где $\Theta(\lambda)$ - функция Хевисайда. Воспользовавшись тем, что $\delta / T \rightarrow \infty$, мы приходим к выражению

$$
\begin{aligned}
J_{q}[\varepsilon] & =\frac{T^{2} f(q)}{2 \varepsilon_{0}^{\prime}}\left(\varepsilon_{1}(q)-\frac{\delta \varepsilon_{0}^{\prime}}{T}\right)^{2}+\frac{T^{2} f(q)}{\varepsilon_{0}^{\prime}} \int_{-\infty}^{\infty}\left[\ln \left(1+e^{-\mu}\right)+\mu \Theta(-\mu)\right] d \mu= \\
& =\frac{T^{2} f(q)}{2 \varepsilon_{0}^{\prime}}\left(\varepsilon_{1}(q)-\frac{\delta \varepsilon_{0}^{\prime}}{T}\right)^{2}+\frac{\pi^{2} T^{2} f(q)}{6 \varepsilon_{0}^{\prime}}+\cdots .
\end{aligned}
$$

Аналогично вычисляется степенная поправка от $\delta$-окрестности точки $-q$ :

$$
J_{-q}[\varepsilon]=\frac{T^{2} f(-q)}{2 \varepsilon_{0}^{\prime}}\left(\varepsilon_{1}(-q)-\frac{\delta \varepsilon_{0}^{\prime}}{T}\right)^{2}+\frac{\pi^{2} T^{2} f(-q)}{6 \varepsilon_{0}^{\prime}}+\cdots .
$$

Складывая (П.4), (П.5) с (П.2), мы после несложных преобразований получаем

$$
\begin{aligned}
J[\varepsilon]= & -\int_{-q}^{q} f(\lambda) \varepsilon(\lambda) d \lambda+\frac{T^{2} \pi^{2}}{6 \varepsilon_{0}^{\prime}}(f(q)+f(-q))+ \\
& +\frac{T^{2} f(q) \varepsilon_{1}^{2}(q)}{2 \varepsilon_{0}^{\prime}}+\frac{T^{2} f(-q) \varepsilon_{1}^{2}(-q)}{2 \varepsilon_{0}^{\prime}}+\cdots
\end{aligned}
$$

Точно таким же способом оцениваются интегралы, содержащие функции $u_{i}(\lambda)$,

$$
J\left[u_{i}\right]=T \int_{\mathbb{R}} f(\lambda) \ln \left(1+e^{-u_{i}(\lambda) / T}\right) d \lambda .
$$

Поскольку $u_{i ; 0}(\lambda)=\varepsilon_{0}(\lambda)$, вычисления, полностью аналогичные приведенным выше, приводят нас к следующей оценке:

$$
\begin{aligned}
J\left[u_{i}\right]= & -\int_{-q}^{q} f(\lambda) u_{i}(\lambda) d \lambda+\frac{T^{2} \pi^{2}}{6 \varepsilon_{0}^{\prime}}(f(q)+f(-q))+ \\
& +\frac{T^{2} f(q) u_{i ; 1}^{2}(q)}{2 \varepsilon_{0}^{\prime}}+\frac{T^{2} f(-q) u_{i ; 1}^{2}(-q)}{2 \varepsilon_{0}^{\prime}}+O\left(T^{3}\right) .
\end{aligned}
$$


Благодарности. Работа была выполнена при финансовой поддержке РФФИ (гранты № 11-01-00440_a, 11-01-12037-офи_м-2011), Программы поддержки ведущих научных школ (грант НШ-4612.2012.1) и программы Президиума РАН "Математические методы в нелинейной динамике".

\section{Список литературы}

[1] K. K. Kozlowski, J. M. Maillet, N. A. Slavnov, J. Stat. Mech., 3 (2011), P03018, 38 pp.

[2] K. K. Kozlowski, J. M. Maillet, N. A. Slavnov, J. Stat. Mech., 3 (2011), P03019, 25 pp.

[3] N. Kitanine, K. K. Kozlowski, J. M. Maillet, N. A. Slavnov, V. Terras, J. Stat. Mech., 12 (2011), P12010, 28 pp., arXiv: 1110.0803.

[4] A. A. Belavin, A. M. Polyakov, A. B. Zamolodchikov, Nucl. Phys. B, 241:2 (1984), 333-380.

[5] J. L. Cardy, J. Phys. A, 17:7 (1984), L385-L387.

[6] J. L. Cardy, Nucl. Phys. B, 270:2 (1986), 186-204.

[7] H. Bethe, Z. Phys., 71:3-4 (1931), 205-226.

[8] R. Orbach, Phys. Rev., 112:2 (1958), 309-316.

[9] E. H. Lieb, W. Liniger, Phys. Rev., 130:4 (1963), 1605-1616.

[10] E. H. Lieb, D. C. Mattis, Mathematical Physics in One Dimension, Acad. Press, New York, 1966.

[11] H. J. de Vega, F. Woynarovich, Nucl. Phys. B, 251:3 (1985), 439-456.

[12] H. W. Blöte, J. L. Cardy, M. P. Nightingale, Phys. Rev. Lett., 56:7 (1986), 742-745.

[13] I. Affleck, Phys. Rev. Lett., 56:7 (1986), 745-748.

[14] Н. Боголюбов, А. Изергин, Н. Решетихин, Писъма в ЖЭЭТФ, 44:9 (1986), 405-407.

[15] A. Berkovich, G. Murthy, J. Phys. A, 21:7 (1988), L395-L400.

[16] A. Berkovich, G. Murthy, J. Phys. A, 21:19 (1988), 3703-3721.

[17] A. Klümper, M. Batchelor, J. Phys. A, 23:5 (1990), L189-L195.

[18] A. Klümper, M. Batchelor, P. Pearce, J. Phys. A, 24:13 (1991), 3111-3133.

[19] A. Klümper, Z. Phys. B, 91:4 (1993), 507-519, arXiv: cond-mat/9306019.

[20] A. Klümper, T. Wehner, J. Zittartz, J. Phys. A, 26:12 (1993), 2815-2827.

[21] A. Klümper, "Integrability of quantum chains: Theory and applications to the spin-1/2 $X X Z$ chain", Quantum Magnetism, Lecture Notes in Physics, 645, eds. U. Schollwock, J. Richter, D. J. J. Farnell, R. F. Bishop, Springer, Berlin, 2004, 349-379, arXiv: cond-mat/0502431.

[22] A. Seel, T. Bhattacharyya, F. Göhmann, A. Klümper, J. Stat. Mech., 8 (2007), P08030, $11 \mathrm{pp}$.

[23] C. N. Yang, C. P. Yang, J. Math. Phys., 10:7 (1969), 1115-1122.

[24] C. P. Yang, Phys. Rev. A, 2:1 (1970), 154-157.

[25] M. Takahashi, Prog. Theor. Phys., 46:2 (1971), 401-415.

[26] M. Gaudin, Phys. Rev. Lett., 26:21 (1971), 1301-1304.

[27] Е. К. Склянин, Л. А. Тахтаджян, Л. Д. Фаддеев, ТМФ, 40:2 (1979), 194-220.

[28] L.D. Faddeev, "How algebraic Bethe ansatz works for integrable models", Symétries quantiques (Les Houches, August 1-September 8, 1995), eds. A. Connes, K. Gawedzki, J. Zinn-Justin, North-Holland, Amsterdam, 1998, 149-219.

[29] V.E. Korepin, N. M. Bogoliubov, A. G. Izergin, Quantum Inverse Scattering Method and Correlation Functions, Cambridge Univ. Press, Cambridge, 1993.

[30] Н. М. Боголюбов, В. Е. Корепин, ТМФ, 60:2 (1984), 262-269.

[31] N. M. Bogoliubov, A. G. Izergin, V.E. Korepin, "Quantum inverse scattering method and correlation functions", Exactly Solvable Problems in Condensed Matter and Relativistic Field Theory, Proceedings of the Winter School and International Colloquium (Panchgani, January 30 - February 12, 1985), Lecture Notes in Physics, 242, eds. B. S. Shastry, S. S. Jha, V. Singh, Springer, Berlin, 1985, 220-316. 
[32] J. D. Johnson, B. M. McCoy, Phys. Rev. A, 6:4 (1972), 1613-1626.

[33] L. Mezincescu, R. I. Nepomechie, "Introduction to the thermodynamics of spin chains", Quantum Groups, Integrable Models and Statistical Systems (Kingston, Canada, 13-17 July, 1992), eds. J. LeTourneux, L. Vinet, World Scientific, Singapore, 1993, 168-191, arXiv: hep-th/9212124.

[34] Н. А. Славнов, ТМФ, 121:1 (1999), 117-138.

[35] С. В. Керов, Функи. анализ и его прил., 34:1 (2000), 51-64.

[36] A. Borodin, G. Olshanski, Electron. J. Combin., 7 (2000), R28, 39 pp.

[37] A. Borodin, G. Olshanski, Commun. Math. Phys., 211:2 (2000), 335-358, arXiv: math/9904010.

[38] A. Okounkov, "SL(2) and z-measures", Random Matrix Models and Their Applications, Mathematical Sciences Research Institute Publications, 40, eds. P. Bleher, A. Its, Cambridge Univ. Press, Cambridge, 2001, 407-420.

[39] N. Kitanine, K. K. Kozlowski, J. M. Maillet, N. A. Slavnov, V. Terras, J. Stat. Mech., 5 (2011), P05028, 34 pp.

[40] N. Kitanine, K. K. Kozlowski, J. M. Maillet, N. A. Slavnov, V. Terras, J. Math. Phys., 50:9 (2009), 095209, 24 pp., arXiv: 0903.2916.

[41] N. Kitanine, K. K. Kozlowski, J. M. Maillet, N. A. Slavnov, V. Terras, J. Stat. Mech., 1 (2007), P01022, 17 pp. 